\title{
A Two Element Phased Array Coil Enabling Widespread Application of High Resolution MR Coronary Angiography
}

\author{
Patricia K. Nguyen ${ }^{1}$, Greig Scott ${ }^{2}$, Jan Engvall ${ }^{3}$, Juan M. Santos ${ }^{2}$, Michael V. McConnell ${ }^{1,2}$, \\ Graham Wright ${ }^{4}$, Dwight G. Nishimura ${ }^{2}$, John M. Pauly ${ }^{2}$, Bob S. Hu ${ }^{2}$ and Phillip C. Yang ${ }^{*}, 1,2$ \\ ${ }^{1}$ Stanford University Hospital, Department of Cardiology, Stanford, CA, USA \\ ${ }^{2}$ Stanford University, Department of Electrical Engineering, Stanford, CA, USA \\ ${ }^{3}$ University Hospital, Department of Cardiology Linköping Heart Centre, Linköping, Sweden \\ ${ }^{4}$ University of Toronto, Department of Biophysics, Toronto, Canada
}

\begin{abstract}
Parallel imaging using sophisticated receiver coils has improved the clinical feasibility of magnetic resonance coronary angiography (MRCA). These techniques, however, are not readily available outside advanced imaging centers. Our custom-made 2 -element phased array coil is readily and inexpensively assembled to address this limitation and enable the widespread application of MRCA.

The 2-element phased array coil, comprised of two 4-inch, overlapping circular coils, is specifically designed for MRCA. We compare our prototype coil to two commercially available coils commonly used for MRCA. MRCA has been performed in 14 normal volunteers. Anatomic coverage, image quality, signal-to-noise ratio (SNR), and contrast-to-noise ratio $(\mathrm{CNR})$ are calculated for each coil.

The prototype coil has imaged $92.6 \%(125 / 135)$ of the segments compared to $83.7 \%(113 / 135)$ and $76.3 \%(103 / 135)(p=$ 0.002 ) using the surface coil and cardiac phased array coil, respectively. Excellent or good (grade 1-2) image quality has been attained in $85.9 \%(116 / 135)$ of all the coronary segments using the prototype coil compared to $77.0 \%(104 / 135)$ and $71 \%(96 / 135)$ using the surface and cardiac phased array coils, respectively $(\mathrm{p}=0.025)$. Overall, higher SNR and CNR have been achieved by the prototype coil compared to the surface and the cardiac phased array coils (SNR: $13.5 \pm 5.3 v \mathrm{~s}$ $12.2 \pm 3.7$ vs $9.0 \pm 3.1$ and CNR: $7.5 \pm 5.5$ vs $6.2 \pm 3.3$ vs $3.7 \pm 2.7$, respectively).

Compared to two commercially available coils, the 2-element phased array coil is associated with overall improved SNR and CNR and provides higher image quality with wider anatomic coverage.
\end{abstract}

\section{INTRODUCTION}

Parallel imaging uses spatial imaging acquired from multiple receiver coils to reduce scan time yet provide acceptable image quality and spatial resolution. In magnetic resonance coronary angiography (MRCA), parallel imaging has enabled increased spatial coverage or improved spatial resolution without increasing acquisition time but often at the expense of signal to noise ratio (SNR). The loss of SNR is proportional to the square root of the acceleration factor, which makes application of these techniques in MRCA challenging.

The widespread application of parallel imaging in MRCA, however, is also currently limited to advanced imaging centers. Parallel imaging techniques have required multi-channel RF receivers and multi-element coil systems as well as advanced reconstruction systems and software [1]. Parallel imaging for non-Cartesian (i.e. spiral) reconstructions has been especially challenging. Because

*Address correspondence to this author at the Cardiovascular Medicine, Stanford University School of Medicine, 300 Pasteur Drive, Falk CVRB, Stanford, CA 94305, USA; Tel: (650) 498-8008; Fax: (650) 724-4034;

E-mails: pnguyen@cvmed.stanford.edu, patricia.k.nguyen@gmail.com every pixel contains a contribution from the entire field of view, iterative approaches have been required to remove aliasing artifacts, which is computationally complex and time consuming [2].

Local imaging centers and hospitals which do not have the specialized hardware and software required for parallel imaging, therefore, have relied on commercially available coils (e.g. the 5-inch circular surface coil or the anterior element of the rectangular cardiac phased array coil) for coronary artery imaging. These coils are general purpose coils for imaging moderate sized structures and are designed to optimize SNR, CNR and other indices of imaging quality for these larger structures. In general, these coils can provide reasonable assessment of myocardial function and structure but may not be adequate for the assessment of small, tortuous coronary arteries.

The need for more specialized imaging coils in cardiac imaging has been proposed by others and their benefit in cardiac imaging has already been demonstrated [3-6]. Similarly, coronary imaging can be improved by the introduction of a dedicated coronary imaging coil. We have designed and built a 2-element phased array coil specifically for imaging the coronary arteries at $1.5 \mathrm{~T}$ that serves as a low cost alternative to parallel imaging techniques. The dedicated 
coronary coil is comprised of two overlapping circular four inch surface coils. The four inch dimension has been chosen because it approximates the entire depth of the heart and minimizes imaging of non-cardiac structures. The width and length of the two combined elements approximate the dimensions of the anterior surface of the heart. Because of the simplicity of its design and components, this coil can be readily and inexpensively assembled and applied for coronary artery imaging. The 2-element coil does not require specific software changes to display a composite image from both elements although a specialized imaging platform (e.g. the dynamic real time architecture) is required for display of images from each element. We compare the imaging performance of the 2-element phased array coil with the 5inch surface coil and the cardiac phased array coil (i.e. anterior element only composed of 2 rectangular elements) in 14 normal volunteers.

\section{MATERIALS AND METHODOLOGY}

\section{Coil Design}

A 2-element phased array receiver coil has been designed and constructed using the shape and dimensions that are suitable for imaging the heart of a normal adult (Fig. 1a, b). Each coil element has been built from copper tape to form a circular shape 4 inches in diameter. The diameter of each coil has been chosen to approximate the depth of the heart, while minimizing coverage of non-cardiac structures; thus, a posterior element is not required. The overall dimension of the two overlapping coils approximates the anterior surface of the heart. The two elements are mounted on a Plexi-glass support. To eliminate mutual conductance, the 4-inch coils are overlapped by approximately 1 inch which has been determined empirically. The total dimensions of coil (mounted on the Plexi-glass support) are approximately $8.7 \mathrm{x}$ 7 inches. Decoupling has been optimized by measuring the cross talk between the coils at different geometric overlaps using a network analyzer (Hewlett-Packard 3589A).

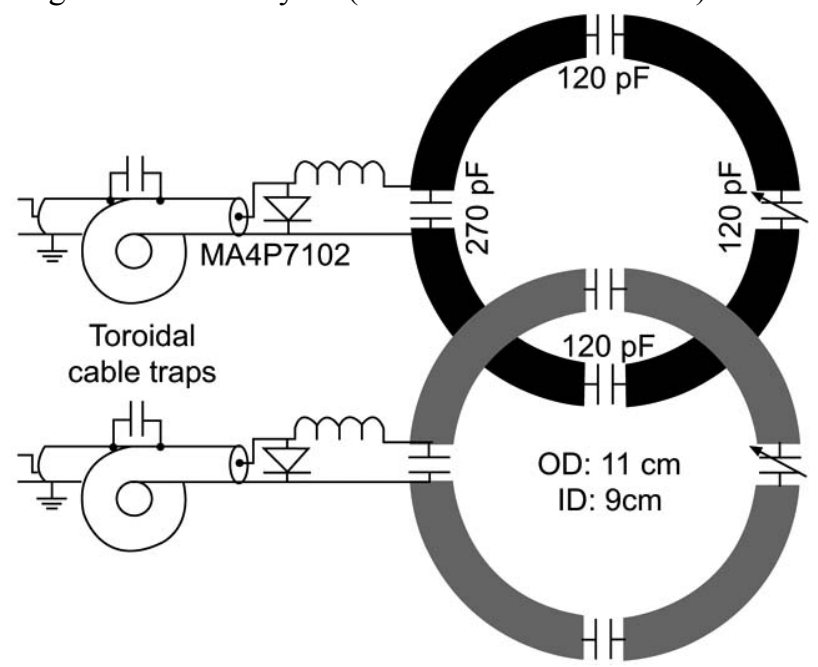

Fig. (1a). Schematic diagram of the 2-element phased array coil. The 2-element phased array coil is composed of two overlapping circular coils, each four inches in diameter. The size of each element has been chosen to approximate the anterior surface of the heart while providing adequate penetration to image posterior coronary artery segments.

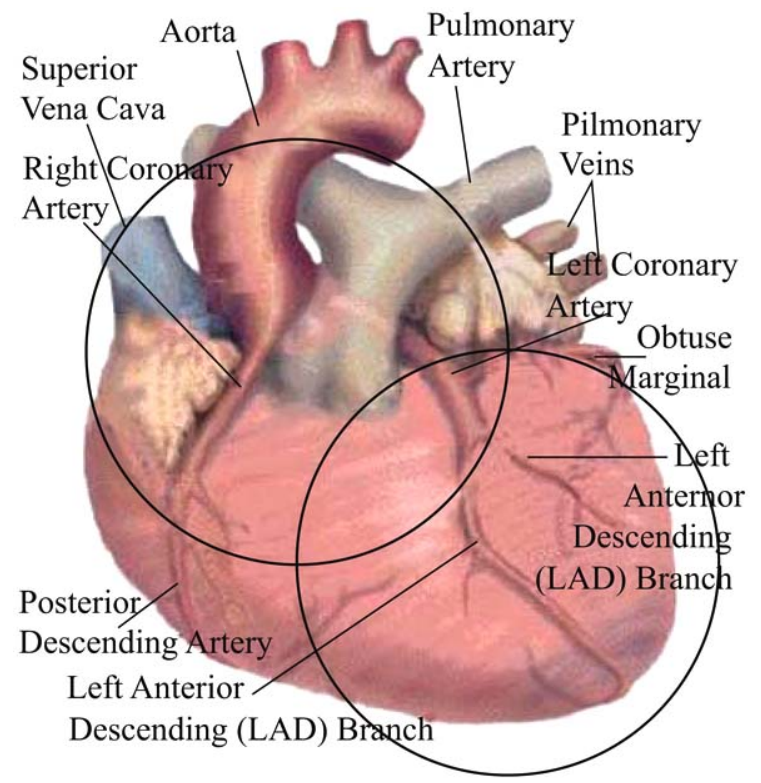

Fig. (1b). Schematic diagram showing the placement of the 2element phased array coil on the anterior surface of the heart. The proximal coil covers the left main, proximal left anterior descending artery, left circumflex and right coronary artery. The distal coil covers the mid- and distal-LAD.

Since coil loading is dominated by the electrical conductivity of the human body, no additional efforts have been made to improve the unloaded quality factor (Q factor) of the coils. Within a certain volume above and below the overlapping region of the 2-element surface coil assembly, the resultant receiver RF magnetic field is the superposition of the two elements, allowing for improved sensitivity. The coil dimensions have been chosen so that the center of the coil sensitivity volume encompassing most of the heart is at the center of the myocardium [7]. To reduce RF electric field interactions and, thus, improve the loaded $\mathrm{Q}$ factor of the array coil, each coil element is split into 4 sections connected in series by distributed capacitors, one of which is used for tuning the coil resonance frequency to $63.9 \mathrm{MHz}$. Matching the impedance of the coil array to the $50 \Omega$ impedance of the cable has been achieved by a tunable capacitive coupling network, followed by a cable shield trap to avoid unwanted shield currents. The surface coils are detuned from the whole body coil during transmission by PIN diode blocking.

\section{Imaging Protocol}

The study protocol has been approved by the Human Subjects Committee at Stanford University. All participants have given written informed consent. MR imaging is performed in the supine position. A scout scan was initially performed to ensure the proper location of the coils over the heart. As detailed below, the protocol consists of real time (RT) localization of the desired coronary segment immediately followed by breath-held (16 heart beats), cardiac-gated high resolution (HR) spiral imaging (using a plethysmogram). The same real time localization and high resolution system (e.g. dynamic real-time architecture) was used for image acquisition for all three coils. Three slices, each 5-mm thick, are obtained with each breath-hold. For the 2-element phased array coil, three different sets of images 
are acquired. One set for each element and a third set are formed from the composite of the two coils. Only the composite image is used for analysis. In contrast, the surface and cardiac phased array coils produce one set of images. When further optimization of the image quality is needed in a specific coronary segment (e.g. respiratory artifact, improper gating, incorrect scan plan location in high resolution scan), the protocol is repeated at most 5 times for each coil. If the segment is not visualized after 5 acquisitions with adjustments made to the imaging plane or breath-hold position, the segment was considered missing.

\section{MR Imaging System}

\section{MR Scanner}

All experiments have been conducted on a 1.5 Tesla $\mathrm{CV} / \mathrm{I}$ whole body scanner (GE Sign Twin, General Electric, Milwaukee, WI) with high performance gradient $(40 \mathrm{mT} / \mathrm{m}$ peak amplitude and $150 \mathrm{mT} / \mathrm{m} / \mathrm{msec}$ slew rate), equipped with four independent broadband receiver channels.

\section{Dynamic Real - Time Architecture (dRT)}

A detailed description of $d$ RT has been reported by Yang et al. [8]. Briefly, the $d \mathrm{RT}$ enables reconfiguration of a pulse sequence within a scan repetition time (TR). A modern PC (AMD, Santa Clara, CA) schedules the imaging sequence and calculates the waveform to generate a scan protocol and pulse sequence on the fly. This capability has been implemented into a real-time imaging environment and enables interactive selection of a desired imaging sequence due to simultaneous sharing of data and resources among different sequences. The $d \mathrm{RT}$ images the coronary arteries through a rapid switch between RT localization and cardiacgated, multi-slice HR spiral imaging sequences [9-11] as shown in Fig. (2a, b). The RT and HR sequences share the

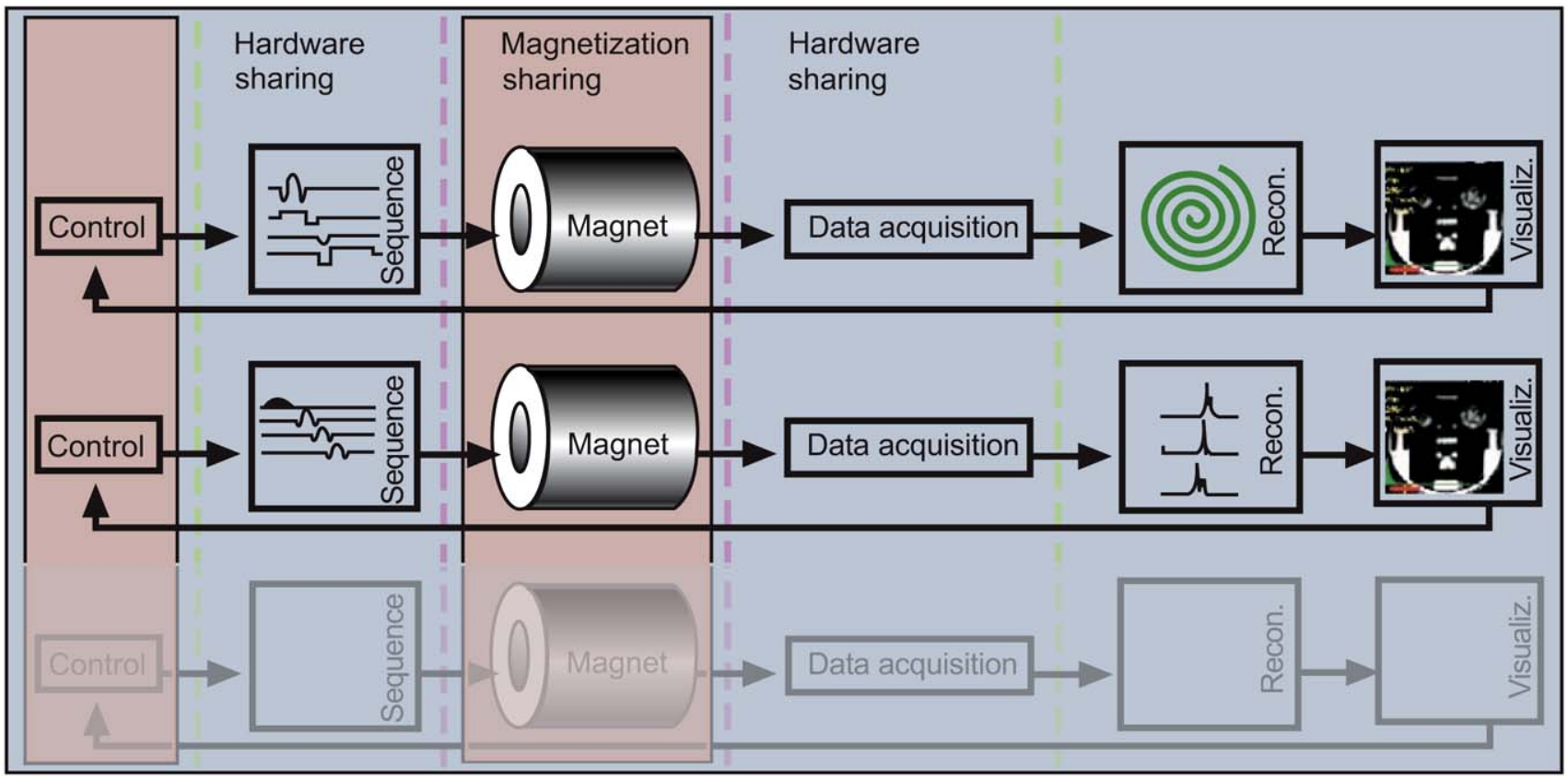

Fig. (2a). Schematic diagram of the Dynamic Real Time $(d \mathrm{RT})$ system. The real time and high resonance sequences share the same spectralspatial pulse and spiral read-out gradient, generating identical TR and FOV. The different variables are the number of spiral interleaves, echo time, flip angle and the resultant spatial resolution.

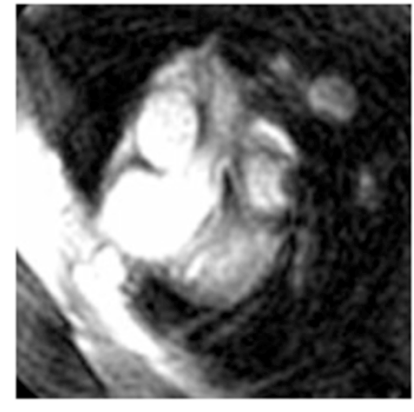

Real time localization
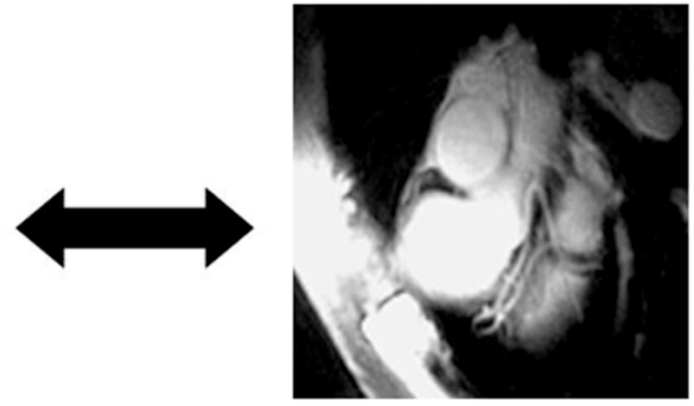

\section{Breath held high resolution} spiral acquisition

Fig. (2b). The $d$ RT images the coronary arteries through a rapid switch between RT localization and cardiac-gated, multi-slice HR imaging sequences. This enables scan plane adjustment on the fly if necessary to improve localization. 
same spectral-spatial pulse with time duration of 11-ms and spiral read-out gradient with time duration of $16-\mathrm{ms}$ and spiral readout of 4096 data points per interleaf, generating identical TR $=39 \mathrm{~ms}$ and field of view $(\mathrm{FOV})=20 \mathrm{~cm}$. The different variables are the number of spiral interleaves, echo time (TE), flip angle (FA), and the resultant spatial resolution. The RT utilizes 3 interleaves with $\mathrm{TE}=4.9 \mathrm{~ms}$ and $\mathrm{FA}=30^{\circ}$ to generate $1.9 \mathrm{~mm}$ spatial resolution. The $\mathrm{HR}$ implements 16 interleaves with $\mathrm{TE}=6.9 \mathrm{~ms}$ and $\mathrm{FA}=60^{\circ}$ and achieves an in-plane spatial resolution of $0.72 \mathrm{~mm}$. Table 1 summarizes the imaging parameters for real time and high resolution imaging.

Table 1. Imaging Parameters for DRT

\begin{tabular}{|l|c|c|}
\hline & $\begin{array}{c}\text { Real Time } \\
\text { Sequence }\end{array}$ & $\begin{array}{c}\text { High Resolution } \\
\text { Sequence }\end{array}$ \\
\hline \hline Spectral spatial pulse & $11 \mathrm{~ms}$ & $11 \mathrm{~ms}$ \\
\hline Spiral read-out duration & $16 \mathrm{~ms}$ & $16 \mathrm{~ms}$ \\
\hline FOV & $20 \mathrm{~cm}$ & $20 \mathrm{~cm}$ \\
\hline TR & $39 \mathrm{~ms}$ & $39 \mathrm{~ms}$ \\
\hline TE & $4.9 \mathrm{~ms}$ & 6.9 \\
\hline Flip angle & $30^{\circ}$ & 16 \\
\hline \# of spiral interleaves & 3 & $0.72 \mathrm{~mm}$ \\
\hline Spatial resolution & $1.9 \mathrm{~mm}$ & $60^{\circ}$ \\
\hline
\end{tabular}

\section{Receiver Coils}

Three different receiver coils have been used to acquire images in all patients. The 2-element circular phased array coil is compared to two commercially available receiver coils commonly used for cardiac imaging: 1) the 5-inch surface coil (General Electric Medical Systems, Milwaukee, WI) and 2) the cardiac phased array coil (anterior element only)(General Electric Medical Systems, Milwaukee, WI). The surface coil is composed of a single, circular coil with a 5 -inch diameter. The cardiac phased array coil is composed of 2 rectangular non overlapping elements measuring $10 \mathrm{x}$ 11 inches. The body coil is used for RF transmission.

The 2-element phased array coil is placed in a cranialcaudal position along the subject's left chest with the right corner of the Plexi-glass at the left sternal clavicular junction. As shown in Fig. (1b), the proximal coil covers the right coronary artery (RCA), left circumflex artery (LCx), left main, and proximal-left anterior descending (LAD). The distal coil covers the mid- and distal-LAD. The 5-inch surface coil was placed along the subject's mid left chest. The cardiac phased array coil is placed in the center of the chest below the chin.

\section{Image Analysis}

All MRCA images have been evaluated independently for coronary coverage and image quality by a total of 3 observers experienced in MRCA and blinded to the coil type and patient identity. Images were displayed in random order of the coil type and the patient identification was removed. If any disagreement occurred, consensus analysis was performed. One observer performed the quantitative measurements of the SNR and CNR. The MRCA image sets are analyzed using the original source images. Images were acquired in similar planes for the major arteries using all three coils. Images of each of the major coronary arteries were displayed sequentially in random order of coil type and patient identity. First, the coverage of coronary anatomy is compared based on the number of coronary segments seen in each coronary artery. The coronary segments are identified according to the American Heart Association classification system $[12,13]$. Side branches are not included. A missing segment was a segment that was not visualized after review of all images acquired for the segment. Second, image quality of each coronary segment is judged using a grading scale based on the extent of the contiguity of the vessel border of a coronary segment (measured in percentage) and the amount of artifact present in the segment (interruption of the vessel border definition). The scale ranges from $1-4(1=$ excellent quality, $>91 \%$ contiguity of the vessel border of a given segment with minimum motion artifact; 2 = good quality, 75$90 \%$ contiguity with minimum to mild motion artifact; $3=$ fair quality, $51-74 \%$ contiguity with minimum to moderate motion artifact; and $4=$ non-diagnostic quality with severe motion artifact or doubling, $<50 \%$ contiguity). Third, SNR and CNR are calculated in all 4 coronary arteries. Blood signal are measured as mean signal intensity of the region of interest (ROI) located within the proximal segment of all 4 coronary arteries. The standard deviation (SD) of the signal intensity of background air is used to estimate the Gaussian noise $(\sigma)$ in the image signal intensity. The noise measurement is adjusted according to the number of elements in the coil [14]. For the 5inch surface coil (one element), the noise in regions with no signal intensity follows a Raleigh distribution and has a SD of $0.655 \sigma$. For the 2 element and cardiac phased array coils (each comprised of two elements), the noise has a SD of $0.682 \sigma$. The myocardial signal is measured as mean signal intensity of the ROI within the left ventricular wall. The SNR and CNR is calculated using the following formulas:

$\mathrm{SNR}=$ Blood signal $/ \mathrm{SD}$ of noise
$\mathrm{CNR}=($ Blood signal - Myocardial signal $) / \mathrm{SD}$ of noise

\section{Statistical Analysis}

The image quality for each coronary segment is expressed as the mean $\pm \mathrm{SD}$. Cohen's Kappa is calculated to assess inter-observer agreement of image quality between two individual readers. A Kappa value of 0.80 to 1.00 is considered very good agreement [15]. A Chi square test ( $\mathrm{df}$ $=2, \alpha=0.05$ ) is performed to determine the presence of significant differences in the anatomical coverage and image quality. Analysis of variance (ANOVA) followed by post hoc Fisher exact test is performed to determine the presence of significant differences in SNR and CNR among the three different coils. If adequate coverage is only possible for two coils in a particular coronary segment, a paired Student's t test is performed (two tailed, $\alpha=0.05$ ). All statistical analyses are performed with StatView (Version 5, SAS Institute Inc., Cary, NC).

\section{RESULTS}

A total of 14 volunteers ( 9 men, 5 women; mean age 39.1 \pm 8.8 years) have been recruited consecutively. All subjects have completed the study without complications. Although 
there is a trend favoring shorter scan time using the 2element phased array coil, this is not statistically significant. Mean scan time for the 2-element phased array, 5-inch surface and cardiac phased array coils are $28.7 \pm 10.3,32.8 \pm$ 12.3 , and $31.1 \pm 10$ minutes $(p=0.75)$, respectively. Scan times are variable per patient and per coil type because acquisitions are repeated if significant respiratory or motion artifact is present. The same reconstruction sequence is used for all patients and all coil types.

Three investigators have analyzed the images. There is minimal variation among the three observers. The Kappa score (linear weighted) between observer 1 and 2, observer 1 and 3 and observer 2 and 3 are 0.95 (SE 0.01; 95\% CI 0.930.97), 0.97 (SE 0.01; 95\% CI 0.96-0.99), 0.97 (SE 0.01; 95\% CI 0.95-0.98), respectively.

Table 2 details the mean and percentage of coronary segments seen in each coronary artery using the three different coils. The 2-element phased array coil has imaged $92.6 \%$ $(125 / 135)$ of the segments compared to $83.7 \%(113 / 135)$ and
$76.3 \%(103 / 135)(\mathrm{p}=0.002)$ using the 5-inch surface coil and cardiac phased array coil, respectively. The 2-element phased array coil provides significantly better coverage of the distal LAD $(p=0.01)$. Improved coverage of the distal RCA and distal LCX is also found but such coverage is not statistically significant.

Table $\mathbf{3}$ details the average image quality in each coronary segment using the three different coils. Excellent or good (grade $1-2)$ image quality has been obtained in $85.9 \%(116 / 135)$ of all the coronary segments using 2-element phased array coil compared to $77.0 \%(104 / 135)$ and $71.0 \%(96 / 135)$ using the surface coil and the cardiac phased array coil, respectively ( $\mathrm{p}=$ 0.025 ). Although not statistically significant, the prototype coil provides better image quality in distal coronary segments and posterior segments yet preserved image quality in the proximal segments. Overall, the use of the 2-element phased array coil is associated with improved image quality compared to the surface (ANOVA $p=0.001,1.57 \pm 1.09$ vs $2.11 \pm 1.40$, Fisher exact $p$ $=0.001)$ and the cardiac phased array coils $(1.57 \pm 1.09$ vs $2.4 \pm$ 1.55 , Fisher exact $\mathrm{p}<0.001)$.

Table 2. Comparison of Anatomic Coverage by Coronary Segment Achieved by the Three Coils

\begin{tabular}{|c|c|c|c|c|}
\hline & 2 Element Phased Array & 5 Inch Surface Coil & Cardiac Phased Array & p Value \\
\hline \hline Left main & $100 \%(15 / 15)$ & $100 \%(15 / 15)$ & $86.7 \%(13 / 15)$ & NS $(0.97)$ \\
\hline pLAD & $100 \%(15 / 15)$ & $100 \%(15 / 15)$ & $100 \%(15 / 15)$ & NS $(0.55)$ \\
\hline mLAD & $100 \%(15 / 15)$ & $100 \%(15 / 15)$ & $93.3 \%(14 / 15)$ & NS $(0.88)$ \\
\hline dLAD & $93.3 \%(14 / 15)$ & $46.7 \%(7 / 15)$ & $33.3 \%(5 / 15)$ & $0.009 *, 0.001{ }^{\circ}$ \\
\hline pRCA & $100 \%(15 / 15)$ & $100 \%(15 / 15)$ & $100 \%(15 / 15)$ & NS $(0.97)$ \\
\hline mRCA & $100 \%(15 / 15)$ & $100 \%(15 / 15)$ & $93.3 \%(14 / 15)$ & NS $(0.88)$ \\
\hline dRCA & $86.7 \%(13 / 15)$ & $66.7 \%(10 / 15)$ & $66.7 \%(10 / 15)$ & NS $(0.13)$ \\
\hline pLCx & $100 \%(15 / 15)$ & $100 \%(15 / 15)$ & $93.3 \%(14 / 15)$ & NS $(0.88)$ \\
\hline dLCx & $66.7 \%(10 / 15)$ & $53.3 \%(8 / 15)$ & $26.7 \%(4 / 15)$ & NS $(0.18)$ \\
\hline Overall & $92.6 \%(125 / 135)$ & $83.7 \%(113 / 135)$ & $76.3 \%(103 / 135)$ & $0.002 *,<0.0001$ \\
\hline
\end{tabular}

LAD: left anterior descending artery; RCA: right coronary artery; LCx: left circumflex artery; p: proximal; m: mid; d: distal

*5 inch surface coil.

${ }^{\circ}$ Cardiac phased array.

Table 3. Comparison of Image Quality by Coronary Segment Achieved by the Three Coils

\begin{tabular}{|c|c|c|c|c|}
\hline & 2 Element Phased Array & 5 Inch Surface Coil & Cardiac Phased Array & p Value \\
\hline \hline Lmain & $1.33(0.62)$ & $1.13(0.35)$ & $2.2(1.27)$ & 0.63 \\
\hline pLAD & $1.07(0.26)$ & $1.18(0.38)$ & $1.42(0.50)$ & 0.93 \\
\hline mLAD & $1.09(0.27)$ & $1.51(0.63)$ & $4.84(1.10)$ & 0.72 \\
\hline dLAD & $2.22(1.52)$ & $3.96(1.25)$ & $1.38(0.44)$ & 0.17 \\
\hline pRCA & $1.16(0.34)$ & $1.55(0.53)$ & $1.6(1.08)$ & 0.88 \\
\hline mRCA & $1.11(0.29)$ & $1.47(0.50)$ & $3(1.44)$ & 0.91 \\
\hline dRCA & $1.91(1.45)$ & $2.91(1.74)$ & $1.76(1.17)$ & 0.24 \\
\hline pLCx & $1.53(0.81)$ & $1.6(0.81)$ & $4.47(1.19)$ & 0.99 \\
\hline dLCx & $3.73(1.33)$ & $3.82(1.41)$ & $2.4(1.55)$ & 0.80 \\
\hline Overall & $1.57(1.09)$ & $2.11(1.40)$ & $0.001^{1}$ \\
\hline
\end{tabular}

LAD: left anterior descending artery; RCA: right coronary artery; LCx: left circumflex artery; p: proximal; m: mid; d: distal.

${ }^{1}$ ANOVA $p=0.001$; prototype vs surface coil, Fisher exact $p=0.001$; prototype vs cardiac phased array, Fisher exact $p<0.001$. 


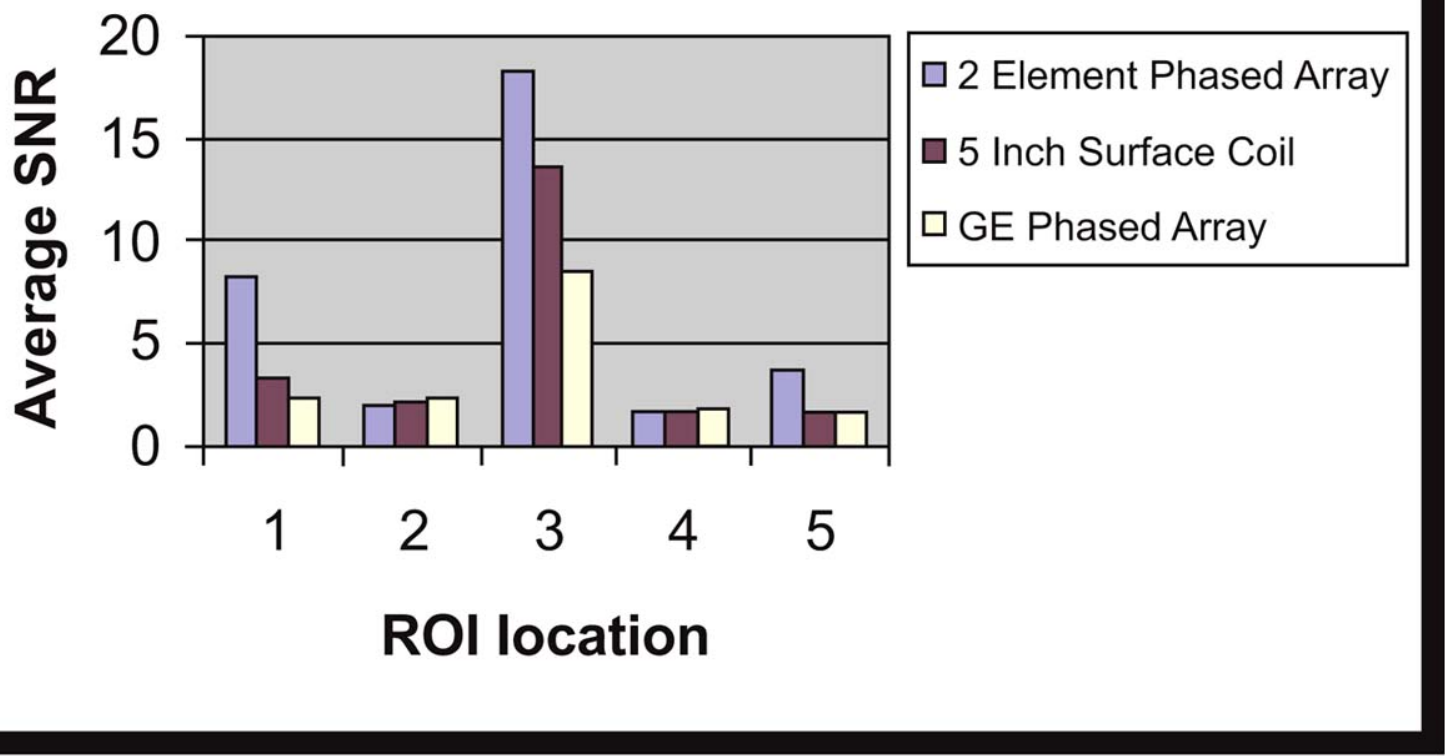

Fig. (3a). The SNR profiles in histogram form from multiple ROI regions for the three different coils. The 2 element phased array coil provides improves SNR in all positions, especially at the center of the image (ROI 3).

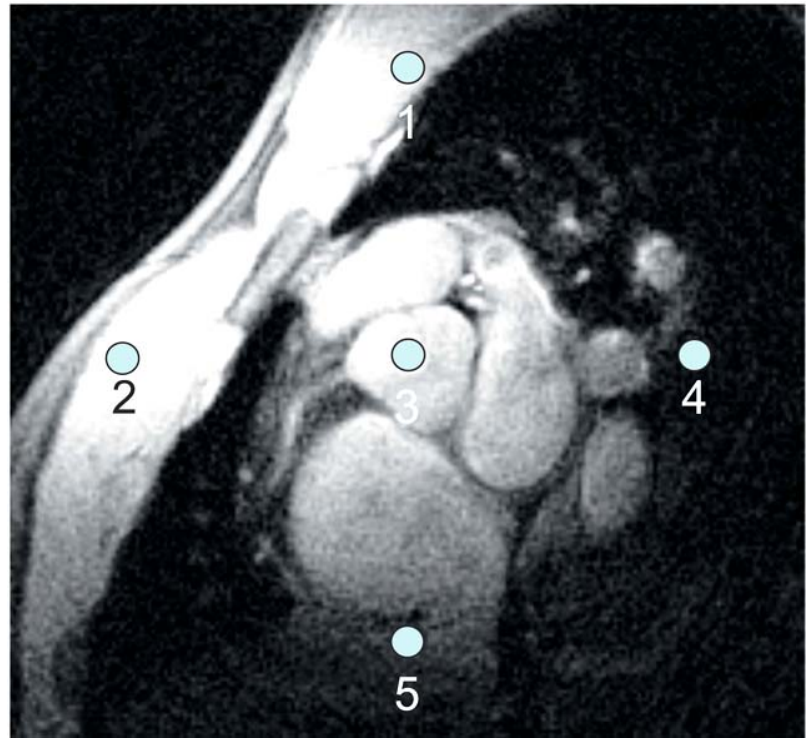

Fig. (3b). High resolution MRCA of the RCA in an oblique view. A region of interest (ROI) was drawn in the center of the image (ROI 3 ) and in 4 peripheral locations as shown.

The SNR profiles for the three different coils are shown in Fig. (3a). A region of interest (ROI) is drawn in the center of an image (ROI 3) of the RCA in the oblique view and in 4 peripheral locations, as shown in Fig. (3b). The average SNR at the center of the image is significantly higher for the 2- element phased array coil compared to the cardiac phased array coil $(21.5 \pm 7.7 v s 10.0 \pm 5.4$, ANOVA $p=0.03$, Fisher exact $p=0.009$ ). Although not statistically significant, the average SNR at the center of the image is higher for the 2 element phased array coil compared to the surface coil (21.5 \pm 7.7 vs $16.7 \pm 10.3$, Fisher exact $\mathrm{p}=0.49$ ).

The 2-element phased array coil has achieved higher average SNR for all coronary segments compared to the surface (Table 4) $(13.5 \pm 5.3$ vs $12.2 \pm 3.7$, ANOVA $\mathrm{p}<0.0001$, Fisher exact $\mathrm{p}<0.001)$ and cardiac phased array coils $(13.5 \pm 5.3$ vs $9.0 \pm 3.1$, Fisher exact $p=0.003)$. Higher SNR is achieved in the left main, mid LAD, proximal RCA, mid RCA, and proximal LCX in both the 2-element phased array and the 5-inch surface coils compared to the anterior element of the cardiac phased array coil. The cardiac phased array coil did not obtain adequate image of the distal LAD and LCX in the majority of patients.

Higher average CNR for all coronary segments has been achieved by the 2-element phased array coil compared to the cardiac phased array coil (Table 5, $7.5 \pm 5.5$ vs $3.7 \pm 2.7$, ANOVA $\mathrm{p}=0.01$, Fisher exact $\mathrm{p}<0.009$ ). There is a trend toward higher overall CNR compared to the 5-inch surface coil but this was not statistically significant $(7.5 \pm 5.5$ vs 6.2 \pm 3.3 , Fisher exact $\mathrm{p}=0.08$ ). Analysis of individual segments reveals that the 2-element phased array coil achieves better CNR in the LM and RCA than the cardiac phased array coil. Comparative images of the LAD and diagonals using the three coils are shown in Fig. (4). 
Table 4. Comparison of SNR Achieved in Each Coronary Segment Using Different Coils

\begin{tabular}{|c|c|c|c|c|}
\hline & 2 Element Phased Array & 5 Inch Surface Coil & Cardiac Phased Array Value \\
\hline \hline Lmain & $21.4(19.3)$ & $14.4(8.0)$ & $10.0(3.0)$ & $<0.001^{1}$ \\
\hline pLAD & $20.7(17.3)$ & $16.0(4.3)$ & 0.15 \\
\hline mLAD & $14.7(5.7)$ & $15.7(5.6)$ & $8.5(3.0)$ & $0.003^{1}$ \\
\hline dLAD & $15.4(7.7)$ & $11.2(5.5)$ & $6.8(2.2)$ & 0.13 \\
\hline pRCA & $17.0(8.2)$ & $13.5(3.4)$ & $5.3(1.9)$ & $0.001^{1}$ \\
\hline mRCA & $10.2(3.8)$ & $12.2(5.8)$ & $7.6(2.6)$ \\
\hline dRCA & $7.6(1.6)$ & $7.7(2.2)$ & $7.4(3.4)$ & $0.004^{1}$ \\
\hline pLCx & $10.8(3.6)$ & $13.0(3.9)$ & $n / \mathrm{a}$ & 0.26 \\
\hline dLCx & $8.2(2.6)$ & $7.4(2.7)$ & $9.0(3.1)$ & 0.31 \\
\hline Overall & $13.5(5.3)$ & $12.2(3.7)$ & $<0.0001^{2}$ \\
\hline
\end{tabular}

LAD: left anterior descending artery; RCA: right coronary artery; LCx: left circumflex artery; p: proximal; m: mid; d: distal

${ }^{1}$ The two element phased array coil had significantly higher SNR than GE phased array coil but not compared to 5 inch surface coil. The 5 inch surface coil also achieved higher SNR than the 4 element phased array coil.

${ }^{2}$ The two element phased array coil achieved significantly higher SNR compared to both the GE phased array coil (Fisher exact $\left.\mathrm{p}<0.001\right)$ and the five inch surface coil (Fisher exact $\mathrm{p}=0.003$ ). The 5 inch surface coil achieved higher SNR than the GE phased array coil (Fisher exact $\mathrm{p}=0.002$ ).

Table 5. Comparison of CNR in Each Coronary Segment Using Different Coils

\begin{tabular}{|c|c|c|c|c|}
\hline & 2 Element Phased Array & 5 Inch Surface Coil & GE Phased Array & P Value \\
\hline Left main & $9.51(6.55)$ & $5.86(4.21)$ & $3.61(2.09)$ & $0.008^{1}$ \\
\hline $\mathrm{RCA}$ & $8.4(3.57)$ & $6.28(2.48)$ & $2.91(1.38)$ & $<0.0001^{1}$ \\
\hline $\mathrm{LCx}$ & $4.1(2.7)$ & $5.68(2.80)$ & $2.69(1.61)$ & $0.01^{2}$ \\
\hline
\end{tabular}

LAD: left anterior descending; RCA: right coronary artery; LCx: left circumflex.

${ }^{1}$ The two element phased array achieved higher CNR in the left main compared to the 5 inch surface coil $(\mathrm{p}<0.05)$ and the GE phased array $(\mathrm{p}<0.05)$.

${ }^{2}$ The surface coil achieved significantly higher CNR than the GE phased array coil (0.003). Although the surface coil achieved higher CNR for the LCx, this was not statistically significant $(\mathrm{p}=0.10)$.

${ }^{3}$ The two element phased array coil achieved higher CNR than the GE phased array coil $(\mathrm{p}=<0.009)$. There was a trend higher CNR was achieved compared to the surface coil but did not reach $(0.08)$. The surface coil achieved higher CNR than the GE $(<0.001)$.

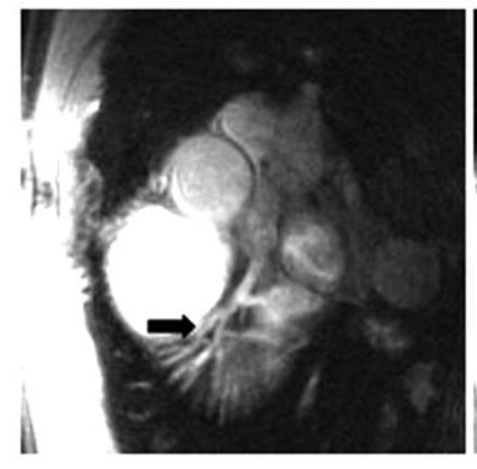

a

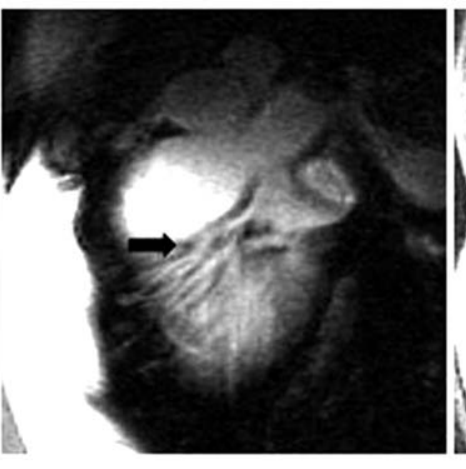

b

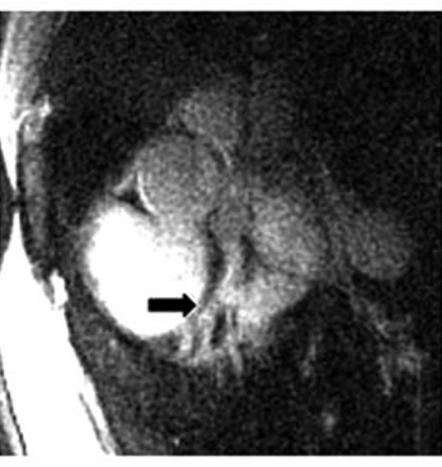

C

Fig. (4). Spiral high resolution images of the left coronary system using the 3 coils. There is improved SNR in the 2-element phased array coil (a) compared to the surface coil (b) and cardiac phased array coil (c), resulting in better delineation of the left anterior descending artery.

\section{DISCUSSION}

Although parallel imaging utilizing sophisticated receiver coils has improved the clinical feasibility of MRCA, its implementation can be costly and is not readily available in all centers. We present an alternative strategy: an inexpensive, easy to assemble 2-element phased array coil for coronary artery imaging. The 2 element phased array coil 
has achieved a percent improvement in SNR of $11 \%$ and $50 \%$ compared to the surface and cardiac phased array coils, respectively. The percent improvement in CNR is $21 \%$ and $105 \%$ compared to the surface and cardiac phased array coils, respectively. The improvement in overall SNR and CNR has resulted in better anatomic coverage and image quality compared to the two commercially available coils.

The 2-element phased array coil is composed of two surface coils. Surface coils have provided improved enhancement in SNR relative to traditional whole volume coils [7, 16-18]. The geometry of the surface coils has been chosen to maximize the SNR for a particular region of interest in the sample [3]. Currently available surface coils are not optimized for coronary imaging and a more extensive sensitive region is often sought to image larger cardiac structures, resulting in larger surface areas and inferior SNR performance for coronary artery imaging. In our study, we found that the SNR and image quality tended to be lower with the 5-inch surface coil compared to the 2-element phased array coil possibly due to a more than adequate penetration of posterior segments. Furthermore, more limited anatomic coverage is achieved by the 5-inch surface coil because the surface area of both the right and left coronary tree often extends beyond the field of view provided by the coil.

The limitations of surface coils have been addressed by the development of phased array surface receiver coils [3, 19]. These coils can permit the best SNR of an individual coil in an array, essentially over the entire extent of the array, thereby providing single surface coil performance with the extended field of view of a volume coil. In addition, the presence of multiple coils with different mutual phase relationships provides an approximate $\sqrt{2}$ increase in SNR which cannot be achieved with a lone surface coil $[16,18$, 19]. Thus, phased array coils produce higher image spatial resolution by increasing SNR while providing a larger FOV coverage [19].

Accordingly, phased array coils consisting of two to four elements have been shown to improve image quality in cardiac imaging $[3,5]$. A recent study has found that the optimum design of phased array coils for cardiac imaging at $1.5 \mathrm{~T}$ is a linear configuration, which outperformed a cluster configuration due to less overlap and decreased coil interactions and noise correlations [3]. The addition of coils far from the heart does not improve image quality and produces diminishing returns in myocardial MR sensitivity. In addition, the use of two coil pairs, one in the front and one on the back, does not significantly improve sensitivity of the heart compared to placing a single pair on the chest $[3,5]$.

The coronary coil is a phased array coil, consisting of 2 elements, both 4 inches in diameter, positioned in an overlapping linear configuration on the anterior chest wall. This provides adequate penetration for imaging posterior segments as well as a FOV which covers both the right and left coronary system, thus, confirming that a posterior element is not needed. More importantly, the 4-inch diameter per coil element provides higher SNR and CNR over the target imaging area (myocardial surface covering the trajectories of the coronary arteries) when compared to the 5inch surface and the cardiac phased array coil.
Moreover, in our study, both the 2-element phased array and the surface coils have outperformed the cardiac phased array coil, composed of 2 rectangular non overlapping elements measuring $10 \times 11$ inches arranged in a linear configuration. The cardiac phased array coil has achieved lower SNR and CNR mainly due to its larger FOV which may be ideal for myocardial imaging but often impairs coronary imaging. Similarly, a recent study [5] has reported inferior SNR for phased array coils with larger FOV. The larger FOV, however, means that the optimum performance of the coil is less position dependent [3], which may be a potential limitation of both the 2-element phased array and surface coils.

A major limitation of this study is the small sample size. Although the 2-element phased array coil has shown improvement in the SNR, CNR and image quality, this did not reach statistical significance in all coronary segments. The study may have limited power to detect these differences. In addition, a less than ideal placement of the coil and the prototype nature of the coil may have also contributed to the variability in SNR, CNR and image quality and reduced significance. A second limitation of the study is that a comparison to parallel imaging techniques was not performed. The primary objective of the study, however, is to demonstrate that the 2-element phased array coil is a low cost alternative that can provide improved image quality and wider anatomic coverage than commercially available coils for centers not equipped with parallel imaging capabilities and/or those without sophisticated receiver coils. Further study is required to show its advantages or limitations compared to parallel imaging techniques.

\section{CONCLUSION}

The utility of a prototype phased array receiver coil suitable for coronary imaging on a clinical 1.5T GE scanner has been demonstrated. This design provides improved anatomic coverage, image quality and overall SNR and CNR compared to two commercially available coils. The findings from this study suggest that the development of inexpensive yet effective receiver coils dedicated to coronary artery imaging may be an alternative strategy for wider application of MRCA in centers who are not yet equipped with parallel imaging techniques or do not have sophisticated receiver coils.

\section{CONFLICTS OF INTERESTS}

There are no conflicts of interests, specifically, there is no financial relationship with the authors and the 2 element coil.

\section{REFERENCES}

[1] Glockner JF, Hu HH, Stanley DW, Angelos L, King K. Parallel MR imaging: a user's guide. Radiographics 2005; 25(5): 1279-97.

[2] Heberlein $\mathrm{K}, \mathrm{Hu} \mathrm{X}$. Auto-calibrated parallel spiral imaging. Magn Reson Med 2006; 55(3): 619-25.

[3] Bottomley PA, Lugo Olivieri $\mathrm{CH}$, Giaquinto $\mathrm{R}$. What is the optimum phased array coil design for cardiac and torso magnetic resonance? Magn Reson Med 1997; 37(4): 591-9.

[4] Constantinides CD, Westgate CR, O'Dell WG, Zerhouni EA, McVeigh ER. A phased array coil for human cardiac imaging. Magn Reson Med 1995; 34(1): 92-8. 
[5] Noeske R, Seifert F, Rhein KH, Rinneberg H. Human cardiac imaging at $3 \mathrm{~T}$ using phased array coils. Magn Reson Med 2000; 44(6): 978-82

[6] Weiger M, Pruessmann KP, Leussler C, Roschmann P, Boesiger P. Specific coil design for SENSE: a six-element cardiac array. Magn Reson Med 2001; 45(3): 495-504.

[7] Edelstein WA, Schenck JF, Hart HR, Hardy CJ, Foster TH, Bottomley PA. Surface coil magnetic resonance imaging. JAMA $1985 ; 253(6): 828$

[8] Yang PC, Santos JM, Nguyen PK, et al. Dynamic real-time architecture in magnetic resonance coronary angiography-a prospective clinical trial. J Cardiovasc Magn Reson 2004; 6(4): 885-94.

[9] Meyer CH, Hu BS, Nishimura DG, Macovski A. Fast spiral coronary artery imaging. Magn Reson Med 1992; 28(2): 202-13.

[10] Kerr AB, Pauly JM, Hu BS, et al. Real-time interactive MRI on a conventional scanner. Magn Reson Med 1997; 38(3): 355-67.

[11] Yang PC, Meyer CH, Terashima M, et al. Spiral magnetic resonance coronary angiography with rapid real-time localization. J Am Coll Cardiol 2003; 41(7): 1134-41.

[12] Alderman EL, Corley SD, Fisher LD, et al. Five-year angiographic follow-up of factors associated with progression of coronary artery disease in the Coronary Artery Surgery Study (CASS). CASS
Participating Investigators and Staff. J Am Coll Cardiol 1993; 22(4): 1141-54.

[13] Austen WG, Edwards JE, Frye RL, et al. A reporting system on patients evaluated for coronary artery disease. Report of the Ad Hoc Committee for Grading of Coronary Artery Disease, Council on Cardiovascular Surgery, American Heart Association. Circulation 1975; 51(4): 5-40.

[14] Constantinides CD, Atalar E, McVeigh ER. Signal-to-noise measurements in magnitude images from NMR phased arrays. Magn Reson Med 1997; 38(5): 852-7.

[15] Landis JR, Koch GG. An application of hierarchical kappa-type statistics in the assessment of majority agreement among multiple observers. Biometrics 1977; 33(2): 363-74.

[16] Fayad ZA, Connick TJ, Axel L. An improved quadrature or phased-array coil for MR cardiac imaging. Magn Reson Med 1995; 34(2): 186-93.

[17] Axel L. Surface coil magnetic resonance imaging. J Comput Assist Tomogr 1984; 8: 381-4.

[18] Hayes CE, Axel L. Noise performance of surface coils for magnetic resonance imaging at 1.5 T. Med Phys 1985; 12(5): 604-7.

[19] Roemer PB EW, Hayes CE, Souza SP, Mueller OM. The NMR phased array. Magn Reson Med 1990; 16: 192-225.

(C) Nguyen et al.; Licensee Bentham Open.

This is an open access article licensed under the terms of the Creative Commons Attribution Non-Commercial License (http://creativecommons.org/licenses/by$\mathrm{nc} / 3.0 /$ ) which permits unrestricted, non-commercial use, distribution and reproduction in any medium, provided the work is properly cited. 